3 Chapman MAS, Grahn MF, Boyle MA, Hutton M, Rogers J, Williams NS. Butyrate oxidation is impaired in the colonic mucosa of sufferers of quiescent ulcerative colitis. Gut 1994; 35: of quiesc $73-6$.

4 Finnie IA, Taylor BA, Rhodes JM. Ileal and colonic epithelial metabolism in quiescen ulcerative colitis: increased glutamine metabolism in distal colon but no defect in butyrate metabolism. Gut 1993; 34: 1552-8.

\section{Helicobacter pylori eradication and serum pepsinogens}

EDITOR,-We wish to comment on the article by Parente et al (Gut 1995; 37: 210-5) regarding clinical usefulness of functional parameters in duodenal ulcer patients cured for Helicobacter pylori infection.

We agree with the authors about the finding of a fall in pepsinogen I serum concentrations three months after the eradication of the infection and we would like to underline the relevance of such a non-invasive parameter already defined by Samloff as 'gastric mucosa serological biopsy'. ${ }^{1}$ In our experience on 259 (197 $\mathrm{M}$; mean age 52.2 years; range $18-79$ ) duodenal ulcer patients tested two months after $H$ pylori eradication treatment pepsinogen I decreased from mean (SD) $147 \cdot 7(78 \cdot 3)$ to $90.7(49.2) \mu \mathrm{g} / \mathrm{ml}(\mathrm{p}<0.005)$. On the contrary the authors found an increase in pepsinogen I serum concentrations in patients who were not cured, where we found, in the same group, a slight decrease: $145 \cdot 6(80 \cdot 7) v$ $124.6(63.4)(p=N S)$. We believe that this decrease, although not statistically significant, could be explained by the fact that even in the group who were not cured the antibiotic treatment reduces $H$ pylori concentration and consequently the gastric mucosa inflammation. ${ }^{2}$

We also would like to point out that in our experience on the same patient's sample, pepsinogen II (RIA Method, Sorin Biomedica, Saluggia, Italy; normal values $2 \cdot 6-13$ $\mu \mathrm{g} / \mathrm{dl}$ ) decreased dramatically after $H$ pylori infection treatment. Indeed in duodenal ulcer cured patients tested two months after the end of the treatment pepsinogen II decreased from $21 \cdot 6(11 \cdot 8)$ to $8 \cdot 2(4 \cdot 1)(p<0 \cdot 001)$. In agreement with our finding on pepsinogen I even pepsinogen II slightly decreased in patients not cured for $H$ pylori infection $(22 \cdot 7(13 \cdot 6) v 16(7 \cdot 2))$. The possible explanation for these findings is that pepsinogen II is produced mainly by the prepyloric glands where usually the degree of inflammation is higher, because the infection is supposed to start in this gastric area as suggested by the bacterial name.

Using the ROC curves statistical analysis we showed that a decrease in pepsinogen II serum concentrations higher than $38 \%$ was the most sensitive $(75 \cdot 5 \%)$ and specific $(74.5 \%)$ value in predicting $H$ pylori eradication with diagnostic accuracy of $75.2 \%$ among four parameters (gastrin, pepsinogen I, pepsinogen II, and IgG anti-Hp). ${ }^{3}$

Pepsinogen I and II were useful not only to verify the success of an eradication treatment but even in the follow up of cured patients. Indeed in a sample of 70 duodenal ulcer patients tested basally, at two, six, and 12 months after the end of a successful eradication therapy we found: pepsinogen I $151 \cdot 7(71 \cdot 3), \quad 89 \cdot 9(46 \cdot 2), \quad 85 \cdot 6(40 \cdot 7)$, $77 \cdot 3(30 \cdot 6)$; pepsinogen II $21 \cdot 4(12 \cdot 7)$ $8 \cdot 7(4 \cdot 9), 7 \cdot 7(3 \cdot 6), 7 \cdot 3(3 \cdot 4)$.

In conclusion we suggest the use of pepsinogens, especially pepsinogen II, in the clinical monitoring of patients treated for
$H$ pylori infection, as indirect and noninvasive parameters of gastric mucosa inflammation.

F DI MARIO

S KUS

$M$ FERRANA

N DAL BO

Department of Gastroenterology

M PLEBANI

Department of Clinical Biochemistry

M RUGGE

Department of Pathology, University of Padua,
Padua, Italy

1 Samloff IM, Varis K, Ihamaki T. Relationship among serum pepsinogen I, serum pepsinogen II and gastric mucosal histology Gastroenterology 1982; 83: 204-9.

2 Dal Bo' N, Battaglia G, Ferrana M, Salandin S, Vianello F, Savarino V, et al. Gastritis Helicobacter pylori correlated: clinical and morphological modification after eradication World Congress of Gastroenterology, Los Angeles, October 2-7, 1994: 713.

3 Ferrana M, Vianello F, Basso D, Leandro G, Da Bo' N, Salandis S, et al. Serological tests for diagnosis of Helicobacter pylori eradication. Gut 1995; 37 (suppl 2): A195.

\section{Reply}

EDITOR,-We are grateful to Di Mario et a for their letter concerning our article. We are completely in agreement with these authors concerning the fact that serum pepsinogen and pepsinogen II may be regarded as markers of mucosal gastric inflammation in patients with $H$ pylori related gastritis, as their concentrations dramatically decrease after eradication of this bacterium and the resolution of gastritis, as recently shown by ourselves and other authors. ${ }^{12}$

However, we wish to emphasise that serum pepsinogen I and pepsinogen II concentrations may also decline (although not dramatically) after antibiotic treatment in patients where $H$ pylori infection persists, probably as a consequence of reduced $H$ pylori density and gastric mucosal inflammation. We did not see such behaviour in our control group simply because 'our controls' consisted of patients with persistent $H$ pylori infection not receiving any antibiotic regimen but who had been given a short-term regimen with lansoprazole only. Indeed, the gastritis score of these patients, both at three and six months from the end of treatment, unlike cured patients, did not change significantly compared with the pretreatment value.

In contrast, we are not in agreement with Di Mario et al about the usefulness of serum pepsinogen I and pepsinogen II concentrations in clinical practice as reliable indirect markers of successful $H$ pylori eradication, especially in the short-term, for two major reasons: (a) as mentioned above, pepsinogen I and pepsinogen II concentrations may decrease after antibiotic treatment even in patients with persistent $H$ pylori infection, as a consequence of reduced $H$ pylori density and gastric mucosal inflammation. Therefore, the finding of a decline in concentrations of serum pepsinogens immediately after treatment has a low specificity in terms of predicting $H$ pylor eradication; indeed, the diagnostic accuracy of this test reported by Di Mario et al in their own experience is fairly low (75\%); (b) multiple samplings are necessary to record a consisten and persistent decrease in serum pepsinogen and pepsinogen II concentrations related to $H$ pylori eradication (at least the baseline value and those at two and six months after the end of the treatment should be determined). The test is therefore not competitive in terms of cost/accuracy with other non-invasive tests (for example, C13-UBT or C14-UBT), if we consider that, in Italy, each pepsinogen I determination costs roughly the equivalent of f11 (with a double assay for each sample) and pepsinogen II determination is even more expensive, whereas C13-UBT (which is undoubtedly the most accurate non-invasive test for diagnosing $H$ pylori infection) costs $£ 18-25$ depending on the number of tests performed.

To summarise, we believe that serum pepsinogen I and pepsinogen II concentrations are indices of gastric mucosal inflammation in patients with $H$ pylori related gastritis, but, like serum Ig-anti $H$ pylori, they cannot be used in clinical practice as reliable markers of successful $H$ pylori eradication.

F PARENTE G MACONI epartment of Gastro L Sacco University Hospital, Milan, Italy

1 Oderda G, Vaira D, Dell'Olio D, Holton J, Forni $M$, Altare $F$, et al. Serum pepsinogen I and Helicobacter pylori. f Clin Pathol 1990; 43: $762-5$.

2 Hunter FM, Correa P, Fontham E, Ruiz B Sobhan M, Samloff IM. Serum pepsinogens as markers of response to therapy for Helicobacter pylori gastritis. Dig Dis Sci 1993; 38: 2081-6.

\section{BOOK REVIEWS}

Hepatobiliary and Pancreatic Disease. Edited by $\mathrm{H}$ A Pitt, D L Carr-Locke, J T Ferrucci. (Pp 496; illustrated; £95.) Boston: Little, Brown, 1995. ISBN 031-6709-158.

The editors of this comprehensive text, which concerns the diagnosis and management of 42 major hepatic and pancreatic problems, point out in their preface that subspecialisation has now occurred in all of the disciplines involved in the care of patients with hepatobiliary disorders, including surgery, gastroenterology, radiology, and pathology. In this book they have attempted to integrate the opinions of these specialists in discussions of current management and treatment options. The invited authors were asked not to avoid controversies in their chapters but to provide a balanced view of the available options and to arrive at a consensus view of the preferred approaches to patient care. The editors stated in the preface that they believed that with this format the book 'should present a unique contribution to the literature'.

The editors invited 132 specialists to produce a text that is only 486 pages in length The hepatic, biliary, and pancreatic topics are very comprehensive in their range although they mostly exclude reference to paediatric liver disease, which was probably regarded as a subspecialty in its own right. The exception is the inclusion of a chapter on biliary atresia. The care of patients after liver transplantation, which would surely have been of great interest to any aspiring hepatologist, has been omitted as a topic. 